\title{
Modeling of EOG and Electrode Position Optimization for Human-Computer Interface
}

\author{
Niina Nöjd, Jari Hyttinen \\ Department of Biomedical Engineering, Tampere University of Technology \\ P.O. Box 692, FIN-33101, Tampere, Finland \\ +358-3-31152524 \\ niina.nojd@tut.fi
}

\begin{abstract}
The aim of this work was to model electro-oculogram (EOG) to find optimal electrode positions for wearable human-computer interface system. The system is a head cap developed in our institute and with it we can measure EOG and facial electromyography (fEMG) and those can be used to control the computer interface: gaze direction moves the cursor and muscle activations correspond to clicking. In this work a very accurate 3D model of the human head was developed and it was used in the modeling of EOG. The optimal positions of four electrodes on the forehead measuring the vertical and horizontal eye movements were defined. More tests and optimization is still needed to verify the positions. Modeling of EOG and the head model were successful.
\end{abstract}

\section{Keywords}

EOG, volume conductor model, modeling, electrode positions

\section{INTRODUCTION}

In our institute we have constructed a wireless head cap that enables the measurements of facial muscle activations and the movements of the eyes. Our ultimate goal is to develop a humancomputer-interface which could be used without hands: The gaze direction could move the cursor with some facial expressions to correspond clicking. People suffering from severe motor disabilities, due to a variety of causes are often prevented from using normal human-computer interfaces like the mouse and keyboard. The quality of life of these individuals could be improved by providing them with a practical assistive device, with which it's easy to use standard personal computers.

In the head cap the wireless data transmission restricts the number of measurement channels and thus, there are seven measurement electrodes in the head cap. To be able to get a good signal quality with only a few electrodes the electrode positions on the forehead should be planned carefully. In this work a very accurate 3D head model is developed and used to model the EOG. Modeled EOG is

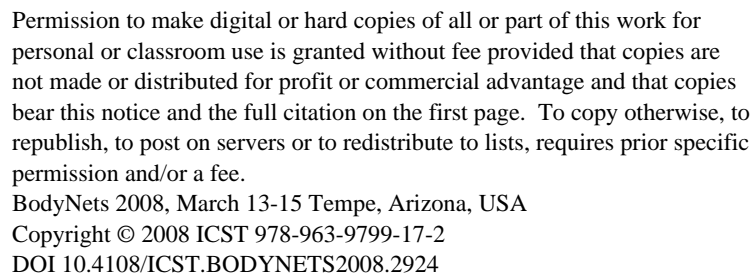

used for optimization of the electrode positions for EOG measurements. Common positions of EOG measurement electrodes are situated on left and right temporals and above and below eye [8]. In our head cap the positions of the measurement electrodes are restrictred to be on the forehead. Modeling is used to find out optimal positions for electrodes there.

Commercial eye-gaze tracking systems are still expensive and techniques used are mainly based on cameras, image processing and following the position of pupil. The use of biosignals like EOG and fEMG is profitable, and along with wireless data transmission and textile electrodes it is possible to design a comfortable measurement device that is easy to use and not confined to some fixed measurement system.

In [4] Joyce et al. have used EOG in eye-gaze tracking. They measured EOG signal, and made a calibration and learning program, in which the gaze was directed into several predefined points in a computer screen, and so the link between signal and gaze was defined. It is true, that mathematical methods such as source localization, solution modeling via optimization constraints, and component decomposition methods are not easily adapted to the eye-gaze tracking, but those solutions should be more studied before disallowing them, and EOG modeling is an important part of that study.

The anatomic accurate 3D model allows us to obtain precise results for the purposes of physiological modeling. Our interest lies in bioelectric forward and inverse problems that enable us to plan optimal electrode configurations for measurement devices and simulate biosignals produced in different organs in body.

\section{MATERIALS AND METHODS}

\subsection{Head Model}

The volume data of the head used was of the Visible Human Female project consisting of anatomical cryosection images and CT slices. The initial segmentation [3] of major tissues was performed employing 3D methods with flexible propagation restraints e.g., 3D active contour and level set methods. The resulting raw segmentations were further fine tuned where necessary using 3D morphological and 2D methods. In some cases when there were very complex structures or almost invisible tissue borders, manual segmentation was the only option. For tissues within the skin and the eyes, synthetic tissue layers were produced using morphological methods due to their low visibility resulting from poor image contrast. 
The volume conductor model included seven different tissue types: scalp, muscle, eye, skull, cerebrospinal fluid (CSF), grey matter, and white matter. The tissue resistivity values that were applied in the volume conductor model are shown in Table 1.

Table 1. Tissue resistivity values.

\begin{tabular}{|c|c|c|}
\hline Tissue & $\begin{array}{c}\text { Resistivity / } \\
\text { Scm }\end{array}$ & Reference \\
\hline Scalp & 351 & $\begin{array}{c}\text { Latikka, Kuurne, Eskola [5] (the } \\
\text { same resistivity than the grey } \\
\text { matter has) }\end{array}$ \\
\hline Muscle & 250 & Duck [1] \\
\hline $\begin{array}{c}\text { Eye (vitreous } \\
\text { humour) }\end{array}$ & 67 & $\begin{array}{c}\text { Oostendorp et al. [7] (resistivity } \\
\text { value of white matter multiplied } \\
\text { by 15) }\end{array}$ \\
\hline Skull & 5265 & Duck [1] \\
\hline CSF & 55 & Latikka, Kuurne, Eskola [5] \\
\hline Grey matter & 351 & [5] \\
\hline White matter & 391 & \begin{tabular}{c} 
Gabriel [2] \\
\hline
\end{tabular}
\end{tabular}

Originally the segmented realistically shaped volume conductor model had a resolution of $0.33 \mathrm{~mm} \times 0.33 \mathrm{~mm}$ x $0.33 \mathrm{~mm}$. Anyhow the resolution had to be decreased in some parts of the model so that the calculation capacity of the computer and the calculation program were able to solve the forward and inverse problems. The best resolution was kept unchanged at the forehead parts, because the eyes, the muscles of interest and the measurement electrodes located there. A sagittal view of the original model and the model with the decreased resolution can be seen in Fig 1.

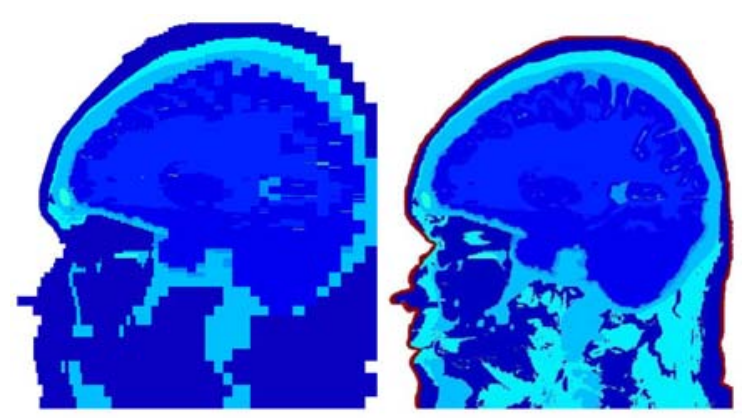

Figure 1. The resolution of the original head model was decreased in posterior and lowest parts of the head. On the left the sagittal view of the model with decreased resolution and on the right of the original model.

\subsection{Simulated EOG}

An iterative FDM solver developed in our institute was used to construct a finite difference method -model (FDM) of the segmented anatomical model. In FDM method the image based head model is discretized into a grid of cubic elements. Elements form a resistor network, where the resistivity of a voxel is defined as the corresponding tissue resistivity and we can insert electrical sources into node points of the resistor network. Reciprocity theorem and lead field concept were used to calculate the surface potentials [6]. Lead vectors were calculated for all the 16804030 nodes in the volume conductor model, and those lead fields were calculated with 33 different surface electrodes, one being all the time a reference.

The lead vectors and source vectors in the eyes were used in the calculations of the surface potentials. EOG source vectors were unit vectors inserted into every node point of the back boundary i.e., the retina of eye balls. The directions of the eye balls in the model were fixed, but source dipole locations were moved to model the changes in the gaze direction. All the source dipoles at the back boundaries were directed parallel to each other, and the direction was chosen to be the same as the gaze direction modeled in each case. This source configuration mimics the charge differential in the eyeball between the interior ends of the photoreceptors and the pigment epithelium in which they are imbedded. Figure 2. clarifies the source model of the EOG used in our simulations.

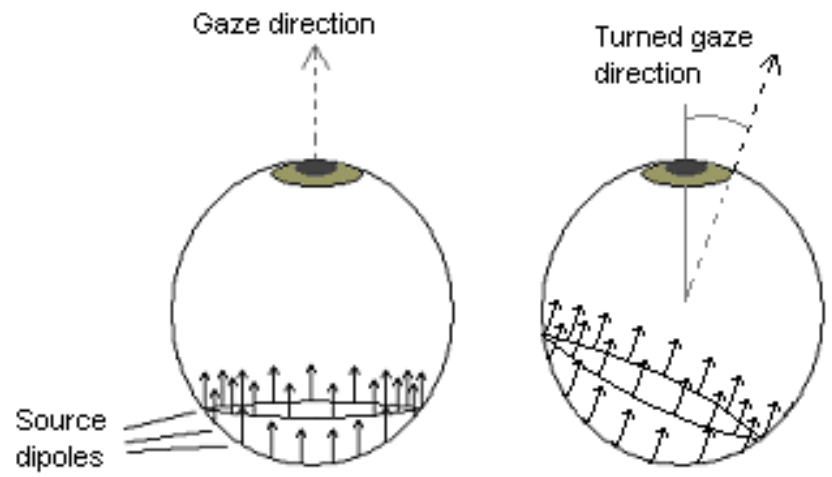

Figure 2. Source dipoles of the EOG signal were on the back boundaries of the eyes. Spherical surfaces were moved to model the movement of gaze direction both in horizontal and vertical directions.

The angle of gaze was changed in $5^{\circ}$ steps from $-60^{\circ}$ to $+60^{\circ}$ (in both vertical and horizontal plane). Altogether 49 different gaze direction and produced surface potentials were modeled.

\subsection{Optimal Electrode Positions}

Wireless data transmission in the current head cap can transfer data from six measurement channels at maximum. Thus the optimum positions for seven measurement electrodes one being the reference should be defined. Three measurement electrodes are needed for corrugator and frontalis muscle activation measurements, and four can be used to measure eye movements. The positions of those four EOG electrodes were defined in this work.

The surface potentials at the forehead were considered on 33 different electrodes. The 49 different gaze directions were treated as independent active sources. Optimal positions for horizontal and vertical eye movement measurements were defined so that one of the sources at a time was chosen to be active and the surface potentials produced by that gaze direction were 
calculated. At first the electrode pairs which produced the biggest potential difference for 49 different gaze directions were defined. It resulted that the optimal electrode positions varied considerably depending on the gaze direction. Therefore the optimal electrode positions were considered on the grounds of how well the electrode pair was able to measure the changes of the gaze directions especially on angles of view between $\pm 20^{\circ}$. This corresponds to eye movements for the human-computer interface where a person is sitting at half a meter distance from the screen.

\section{RESULTS}

The greatest potential values for the horizontally directed angles of views were found from five different electrode pairs (Fig. 3.). The more the gaze was sideward directed the more horizontally positioned was the most sensitive electrode pair. Electrode pairs $(1,32)$ and $(5,32)$ were the most sensitive between the gaze directions $\pm 10^{\circ}$. The same two electrode pairs were the most sensitive for all angles of views in the vertical direction (Fig. 4). Thus, for the little angle of views the electrode pairs were surprisingly the same for the vertical and horizontal gaze directions. Surface potentials where calculated for angles of view between $\pm 60^{\circ}$, but in Fig. 3. only the significant angles for human-computer interface $\left( \pm 20^{\circ}\right)$ are reported.

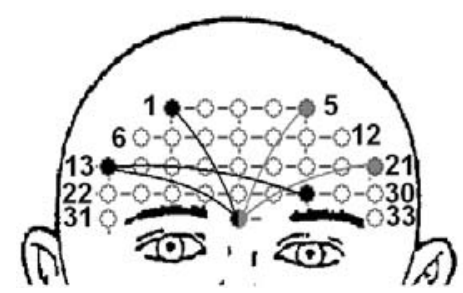

Figure 3. The most sensitive electrode pairs for different angles of view in a horizontal plane (gray electrodes for gazes directed to the right $(+)$ and black ones to the left $(-)$ from a viewer's angle of view). Electrodes are numbered from left to right with numbers $1-33$. The greater the angle of view, the more horizontally the electrode pair was situated. Electrode pairs $(1,32)$ and $(5,32)$ were the most sensitive for the gaze directions $0^{\circ}, \pm 5^{\circ}$ and $\pm 10^{\circ}$, the pairs $(13,28),(13,32)$ for $-15^{\circ}$ and $-20^{\circ}$ respectively, and the pair $(21,32)$ for the gaze directions $+15^{\circ}$ and $+20^{\circ}$.

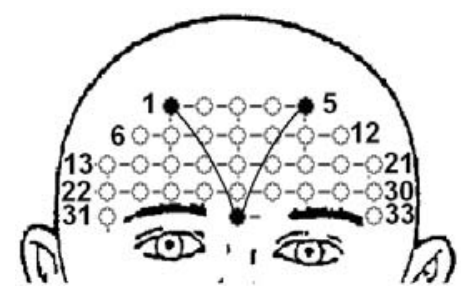

Figure 4. The most sensitive electrode pairs for all angles of view in the vertical direction.

In the human-computer interface the gaze direction is more closely defined. We can use only one electrode pair for vertical and one for horizontal eye movement detection, not all the found electrode positions that were the most sensitive for single gaze directions. Thus we have to define the electrode pair that is most sensitive for the changes of angles of views. We examined which one of the electrode pairs produces the largest potential change with the $\pm 20^{\circ}$ change of the angle of view. In Figure 5 . it can be seen, that for the horizontal and vertical eye movements the greatest potential value change was found almost from the same electrodes, $(29,31)$ and $(28,31)$ respectively.

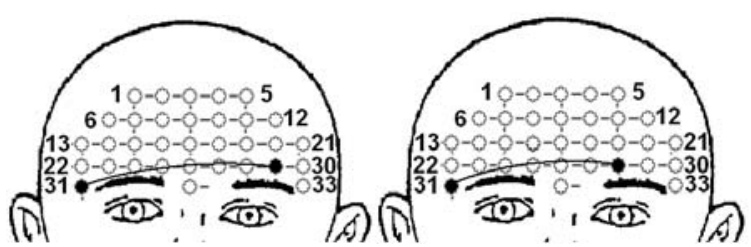

Figure 5. On the left there is the electrode pair, which produced the greatest potential value change between the gaze directions $+20^{\circ}$ and $-20^{\circ}$ in the horizontal eye movement, and on the right the one which produced that in the vertical eye movement.

Our results of the separating capability of small angles show that for the horizontal and vertical gaze directions the best electrode positions would be too similar (Fig. 5.): the overlapping problem of the signals from orthogonal eye movements should be avoided. In the literature [8] it is recommended that for the vertical eye movement detection the electrodes are positioned upright above and below eye. We got the result that the upright positioned electrode pairs $(1,32)$ and $(5,32)$ produced the greatest amplitude for the signal in every vertical gaze direction, but surprisingly the horizontally aligned electrode pair $(28,31)$ was the best for the measurements of the changes of gaze directions. For the vertical gaze directions the potential changes between $\pm 20^{\circ}$ were $0.2094,0.0914$ and 0.0379 for the electrode pairs (28, $31),(1,32),(5,32)$ and the average change of all the possible electrode pairs (altogether 528) was 0.0597. Because the electrode pairs $(1,32)$ and $(5,32)$ lie orthogonally on the forehead compared to the electrode pair $(29,31)$, which was the optimal for the horizontal eye movement, and those measured the vertical eye movements moderately and single stagnant directions best, those were considered to be the best electrodes to measure the vertical direction. Electrode pair $(1,32)$ was more sensitive as the pair $(5$, 32) for the changes in the vertical gaze directions and was thereby selected to be the optimal measurement electrode pair for the vertical gaze directions. The pair $(29,31)$ was the best for the horizontal gaze directions.

\section{CONCLUSIONS}

A new accurate model is now in use for modeling purposes such as bioelectric field problems. It has high spatial accuracy and number of inhomogeneities providing a good platform for various simulations. For the bioelectric simulations with FEM or FDM methods the number of elements in the resulting model exceeds the standard computer resources. The model used in this study had seven but the latest version has already 23 different tissue types.

With the current measurement system the movements of eyes and the activations of two different facial muscles can be measured, and measured information can be used to control the computer interface. We will develop our human-computer system further, 
and modeling of both fEMG and EOG will be used in the development.

In this work the optimal electrode positions for eye movement measurements were defined. Using just the forehead for vertical and horizontal eye movement measurement limits the possible electrode positions and the capability to find optimal locations to separate the horizontal and vertical eye movements. More tests and optimization is still needed.

\section{ACKNOWLEDGMENTS}

The authors would like to acknowledge Markus Hannula and Nathaniel Narra from Ragnar Granit Institute for segmenting the volume conductor model and Noriyuki Takano for the iterative FDM solver.

\section{REFERENCES}

[1] Duck F., Physical Properties of Tissue: A Comprehensive Reference Book, Academic Press, San Diego, 1990.

[2] Gabriel C., Gabriel S., Compilation of the dielectric properties of body tissues at RF and microwave frequencies, 1996.
[3] Hannula M, Narra N, Arola T, Keshvari J, Hyttinen J, Visible Human Female Head Segmentation, Bioelectromagnetics Society 29th Annual Meeting, Kanazawa, Japan, June 10-15, 2007

[4] Joyce C, Gorodnitsky I., King J., and Kutas M., Tracking eye fixations with electroocular and electroencephalographic recordings, Psychophysiology, 2002 , 39: pp. 607-618.

[5] Latikka J., Kuurne T., Eskola H., Conductivity of living intracranial tissues, Physics in Medicine and Biology, vol. 46, pp. 1611-1666, 2001.

[6] Malmivuo J., R. Plonsey, Bioelectromagnetism: Principles and Applications of Bioelectric and Biomagnetic Fields, Oxford University Press, New York, 1995.

[7] Oostendorp T., Delbeke J., Stegeman D., The Conductivity of the Human Skull: Results of In Vivo and In Vitro Measurements, IEEE Transactions on Biomedical Engineering, vol. 47, pp. 1487-1492, 2000.

[8] Thakor, N., The Measurement Instrumentation and Sensors Handbook, CRC Press, 1999 